nating currents. ${ }^{1}$ It differs from the type in not having concave cathodes, in the direction in which the $\mathrm{x}$-light is projected and in the relative positions of the terminals and the target. The two latter changes facilitate inclosing the tube in a nonradiable holder and connecting it with the electric generator, as well as getting the leading wires away from the patient. As the nonradiable tube holders shown in this note do not entirely inclose the tube, the exposed parts require to be covered with nonradiable material or the entire tube, except a small area for the escape of $x$-light, may be treated with an aqueous solution of esculine. NOTE 201. WHY X-LIGHT AFFECTS THE BLOOD
VESSELS.

In 1901 , some of the results of a considerable number of experiments on guinea pigs with $x$-light were briefly reported in this JourNAI. In Note $179 \mathrm{D}$, one of the conclusions reached was advanced as a theory of the action of $x$-light on animal tissues. Since that time the same theory has been advanced by Sir Oliver Lodge. In Note 180, Electrical Review, Jan. 23, 1904, the matter was more fully considered and the theory was used to explain the similar effects of radioactive substances. The theory stated $x$-light and radioactive substances produced ionization of the molecular complexes in the body. The previously known fact of the inability of these agents to affect a daguerreotype plate was mentioned in a later note, and their power to act on the same silver salt when it was mixed with an animal compound, gelatine, in the ordinary dry plate, were placed in contrast; the conclusion being reached that the greater effects on diseased than on normal tissues were due to the larger and less stable aggregations of molecules of the diseased tissues being more easily ionized than those of the normal ones in the same way that the larger molecular complexes of the gelatine dry plate were acted on when the smaller groups of the daguerreotype plate were not. Though in 1901, a pathologist could not be interested to study the guinea pigs injured during my experiments, a considerable number of observers have since examined tissues affected by $x$-light. The general opinion is the blood vessels are more seriously injured than some other tissues. The bearing of this on the ionization theory advanced in these notes is here considered. We know the fluids of the body are passing in and out through the walls of the blood vessels; therefore, some of the ions produced by the action of $x$-light and radioactive substances will be constantly carried by the currents through the cells of the walls of the vessels and their destructive action will there be marked. That ions produced by x-light and radioactive substances exist for some time after their production and can be carried to considerable distances is known to experimenters in physics, and details of a number of investigations may be found in the pages of The Philosophical Magazine during the last few years.

1 Refer to Fig. 4, n. 199.
THREE CASES ILI,USTRATING THE PRACTICAL IMPORTANCE OF RECOGNIZING THE POSTEPILEPTIC STATE.

BY MORTON PRINCF, M.D.,

Physician for Diseases of the Nerrous System, Boston City Hospital.

Tнолан temporary paralysis and various forms of mental delirium following epileptic fits are well recognized, I do not think that the possibility that the paralytic and mental condition may simulate gross lesion of the brain is as fully realized as the practical importance of the matter demands. The post-epileptic state may easily be mistaken for cerebral hemorrhage or tumor, and may seem to call for operative interference, as happened in two of the cases I am about to report.

Sometimes, especially in cerebral syphilis, it may be difficult to determine whether we are dealing with a post-epileptic paralysis, or with a form of syphilitic paralysis. Case II is an illustration of this condition.

Obsenvation I. It is not surprising that this case, in the absence of a complete history, was mistaken for some sort of cerebral hemorrhage, and trephined.

A woman was found unconscious on the floor of her room. Seen by a neurologist and surgeon the question was thought to be between cortical and meningeal hemorrhage. Pupils were "equal, somewhat contracted and responded sluggishly to light." Every ten or fifteen minutes a clonic convulsion occurred involving both sides about equally. There was flaceid paralysis of the left extremity (extremities?) and the left knee jerk was plus. The right extremity (extremities?) spastic and knee jerk present. Babinski present on both sides.

There was no hematoma of the scalp, no bleeding from ears, nose or mouth, although there was an ecchymosis about the left eye. Operation was considered advisable.

On opening the dura there was considerable serous discharge, otherwise nothing remarkable was observed. The next day it was noted the patient continued to have convulsions, but less frequently; that consciousness returned at intervals (between the attacks), and that she moved the left extremities nearly as well as the right. Transferred to the Boston City Hospital. While being taken from the ambulance to the ward the patient had a general convulsion of apparently the classical type. A few minutes later examination again showed evidence of hemiplegia. Both pupils were equal and reacted to light; slight obliteration of the left facial folds, slight drooping of the left corner of the mouth and drawing up of the right corner. Tongue protruded straight. Slight spasticity of right extremities, and moderate flacidity of left. Knee jerk; left slightly exaggerated over the right. Plantar reflex; left Babinski; right, "sluggish."

The patient was conscious and answered questions intelligently, but in a listless and dull manner.

Oct. 5 , it is noticed that the patient's condition is much improved. Mind is much clearer. Examination made yesterday (four days after the attack) showed that the evidence of hemiplegia had entircly disappeared. One convulsion since Oct. 1. Patient states that she has been subject to "fainting spells " ever since she was a small child. On Oct. 13, six days later, it is noted that the patient has had one or two fits each day since last note. At other times perfectly rational No hemiplegia, which was evident on admission. About one week later (Oct. 21), in the interim being well, the 
patient became wildly delirious, talking loudly and attempting to get out of bed; required restraint.

The records do not state the conditions under which this delirium developed, but presumably it followed a convulsion, for it is noted : "Last convulsion yesterday, frothing at the mouth, jaw rigid. Face and body turned to left and then general clonic convulsion, lasting about three minutes. Patient drowsy for some time afterwards."

Oct. 25, I saw the case in consultation and by careful inquiry obtained: First a history of long-standing epilepsy, the attacks being of the grand mal type; second, an account of what was in all probability a convulsion shortly before being found unconscious on the floor of her room. That is to say, the patient gave an account of symptoms similar in all respects to those which always usher in an attack, followed by loss of consciousness.

Physical examination showed no asymmetry, no alteration of the reflexes, no increased myotatic irritability, no muscular weakness, or any of these changes which follow organic hemiplegia even when transient.

In view of the fact that the patient was subject to fits, that she testified that she had had the symptoms which always begin a fit shortly before being found unconscious, that opening the skull showed no hemorrhage, that the hemiplegia nearly completely disappeared on the second day to reappear after a convulsion, and finally disappeared completely and rapidly at some time within four days of the original attack (perhaps carlier), and that after disappearance of the hemiplegia there was no persistent physical sign of increased myotatic irritability such as is usually found following even slight and transient hemiplegias due to organic disease, the diagnosis of post-epileptic paralysis was made.

The patient was put on bromide. The convulsions ccased and three weeks later the patient was discharged " apparently perfectly well mentally," as stated in the record, and none the worse for the surgical treatment.

Observation II. A. B., painter, age thirty-five. Last October he was taken ill in the following way: He began to feel mentally dull, his memory became defective and he experienced a sensation of "buzzing" in his head. The "buzzing " was not continuous, but intermittent and took rather the form of a succession of attacks. (It may be here noted that this subjective symptom was identical with that which he now experiences from time to time and which will be described later.) This condition lasted about one week, during which time he became more and more dull although able to go about, and terminated in a series of convulsions which came on at night when he was in bed and lasted for five days and five nights. Each convulsion lasted from five to ten minutes and left both sides weak but especially the right. At this time he was seen by a neurologist who thought his condition might be due to a cerebral tumor and advised an exploratory operation. At this time, as shown by the record, he was semi-comatose and there was marked paralysis of the whole right side. There was no optic neuritis. $\mathrm{He}$ was put upon $5 \mathrm{gr}$. of iodide and $30 \mathrm{gr}$. of bromide three times a day. At the end of three days the hemiplegia had disappeared and the reflexes were normal. It still being thought a tumor might be present, an operation was performed, the bone being removed over the middle portion of the motor region on the left side. The dura was opened but nothing found though the brain was probed with the little finger for the purpose of sounding for a tumor, and the ventricle tapped with a director without obtaining fluid. Considerable laceration of the cortex resulted. By degrees he recovered from the operation and, syphilis being suspected, he was put upon iodide of potash. There have been no more convulsive attacks, but, what is significant in connection with the history of the prodromes preceding the week of convulsive seizures, he now has frequent attacks of "buzzing," which are without doubt attacks of auras or petit mal. His present condition is that of nearly complete paralysis of the right arm with slight paresis of the face and leg. Otherwise he seems quite well. He is able to walk about but his arm is useless and exhibits a moderate amount of contracture. The deep reflexes on the right side are extremely exaggerated including a marked jaw jerk and ankle clonus. There is no anesthesia; his mind is clear and intelligent. 'There are no occular palsies or implications, of the cerebral nerves such as are frequent in cerebral syphilis; there is no optic neuritis, no headache, dizziness, or nausea, and in fact, with the exception of the monoplegia (or hemiplegia), there is no evidence of tumor, cerebral syphilis, or other organic lesion of the nervous system.

The persistent paralysis seems to be unqestionably due to laceration of the brain occasioned by probing at the time of the operation, and which, according to the statement of the surgeon, to me was considerable.

He now gives the following history of his past life, which corroborates this view: Fifteen years ago he met with an accident by which, owing to the breaking of a rope on board ship, he fell striking his head on the deck. He was unconscious for at least ten days. Seven years later, $i . e$. , eight years ago, he had his first convulsion, in which he lost consciousness. He had no more convulsions until four years ago, when they reappeared and continued in frequency from one a month to three a week. The convulsion always began on the right side, sometimes the initial spasm being in the leg and sometimes in the arm. They were always preceded by the aura of a "buzzing " in the head already described. He met with a second accident Aug. 28, 1903, due to the falling of a stage on which he was standing while painting. The accident was not attended by any ill consequences.

In carefully reviewing this case, and in the light of the history now known, the evidence seems to be conclusive that the paralysis and other cerebral symptoms, for which he was operated upon, were really only post-epileptic phenomena and in the course of time would have spontaneously disappeared, if operation had not been undertaken. It is significant that the patient now recalls and states distinctly that, although he has no recollection of the few days preceding the operation when he was in a semicomatose condition, on the morning of the operation he began to return to himself and could use his right hand.

It seems impossible that there can be any tumor present in view of first: Failure to find any tumor in the arm center, and, second, entire absence of the general symptoms of tumor, and his progressive recovery. As to cerebral syphilis, there is, and has been, no evidence of any focal disease such as would cause monoplegia nor of any diffuse process in the brain or spinal cord. 
It is an interesting fact that in this case, while there was an exquisite ankle clonus on the right, there was no Babinski.

Remarks: The pathology of post-cpileptic paralysis is entirely unknown. It is generally ascribed to exhaustion of the motor centers, but this is purely speculative and has almost nothing in its support. The gush of serum on opening the dura in the first of the above cases suggests the hypothesis that the paralysis may be due to localized edema. Such a pathology would comport with the symptoms, but as yet lacks objective evidence.

Orservation III. The following case, one of post-epileptic delirium (dämmer-zustand) simulating insanity, was admitted to the Boston City Hospital in the medical service. On entrance her condition is described in the records as "apparently one of dementia." No history at the time was obtainable, but shortly afterwards it was learned from her husband that she had been subject to convulsions as long as he had known her, that is, at least for nine years. The attacks occur every three or four weeks and come on suddenly without warning. The patient becomes suddenly unconscious, froths at the mouth, but does not bite the tongue; clonic followed by tonic spasms; head is thrown back and body arched. Rigidity then ceases and the patient awakes as if from sleep. An attack lasts from three to twenty minutes and is usually followed by headaches, but her mind becomes and remains clear.

Fighteen months ago an attack occurred more severe than usual; patient lost her recognition of time and place, became noisy and difficult to manage; sent to Long Island for a week, recovered in ten days.

The present illness began three days before admission to the hospital. She became noisy, talked foolishly, and failed to recognize her surroundings and the time, but not violent. The next day she became stupid and would not answer questions. In reply to every question she kept repeating, "Now I am going to tell you." The patient continued unable to recognize her surroundings, but the stupor and apathy changed to noisiness and she was brought to the Boston City Hospital. On entrance at first "wildly delirious," she later became "semi-unconscious," but responded to painful stimuli; she frequently repeated the stereoptyped phrase, " Now I will tell you." Physical examination was negative. Pupils were of medium size and reacted to light and accommodation.

On Jan. 20, 1904, three days after entrance, I saw the case in consultation with a view of determining whether she should be sent to an asylum. But it seemed to me that the case was plainly one of post-epileptic delirium. This diagnosis was easily made in the light of the history obtained, and was confirmed by the fact that the patient soon, if not suddenly, recovered her senses. When I saw her, while not absolutely clear in mind, and still somewhat confused, she was no longer delirious or demented. In the course of the next day she had a relapse, but this soon clcared up and in a few days the patient was mentally as well as before the attack. The details of the hospital records are incomplete, but it is highly probable that the relapse which occurred followed an epileptic attack, she having had several while in the hospital. After recovery, the patient gave a clear account of herself, stating that she had been subject for years to epileptic attacks, some of which were of the grand mal type, and some of petit mal. The present illness began with an attack which was followed by the above-described niental condition. It did not appear that, with the two ex- ceptions noted, her attacks have been followed by mental delirium. In the hospital records the patient is described as being, after recovery, "very emotional."

This I think can hardly be described as entirely pathological or unreasonable, as the patient had always had an intense dread of becoming insane, and now recovering very rapidly her reason, the poor woman could not make those about her realize that she was not insane, and was in terror of being sent to an asylum.

\section{REMARKS ON THE PRESENT STATUS OF SURGICAL TREATMENT OF HEPATIC CIRRHOSIS.}

By ChaRles GREene cumston, M.D., Boston, Mass.

IN the Berliner klinische Wochenschrift for July 30, 1900, Talma made the following statement: "Insufficiency of the collateral circulation of the blood should be considered as the cause of ascites. The improvement or cure of the affection may result from a gradual development of a collateral circulation. I advise my colleagues, under these circumstances, to open up new avenues of derivation for the blood of the portal vein. I am intending to bring into as close relationship as possible the liver or the large paraumbilical venous system of the abdominal wall in each case coming under observation of atrophie cirrhosis with ascites without disturbances in the cellular function of the liver and I have not been deceived in my expectations." This announcement of the professor of Utrecht did not pass by unnoticed and surgery entered into a new field which had been indicated by medicine. Various operations were performed intended to increase the anastomosis between the portal vein and vena cava in order to drain the distended portal system. Direct cavo-portal anastomosis was proposed and realized, but the operation was far from producing the results hoped for.

For many years hypertension of the portal vein has been considered the cause of ascites and the theories upheld explaining this would be too numerous to mention, but they were pleasing from their very simplicity. The portal vein being obstructed above naturally was thought to become dilated even in its finest branches and from this fact allowed serum to pass through its walls which gives rise to the ascitic fluid. An experimental demonstration of this process was even attempted by Beau as far back as 1851, while Frerichs out of a total of twenty-cight cases of adhesive pylephlebitis observed ascites in twenty-five and in the three other cases he noted abundant gastro-intestinal hemorrhage.

To these mechanical ascites arising from exit of the blood serum from the vessel, inflammatory ascites was opposed. Their manner of appearing, their great ease in reproduction and their composition rendered them very different from inflammatory ascites resulting from peritonitis. Hoppe-Seyler attentively examined the composition of the fluid of ascites arising during 